\title{
Continuous dependence of solutions of abstract generalized linear differential equations with potential converging uniformly with a weight
}

\author{
Giselle Antunes Monteiro ${ }^{1}$ and Milan Trrdý2 ${ }^{*}$ \\ Dedicated to Professor Ivan Kiguradze for his merits in mathematical sciences.
}

"Correspondence:
tvrdy@math.cas.cz
${ }^{2}$ Mathematical Institute, Academy
of Sciences of Czech Republic,
Žitná 25, Praha, 11567, Czech
Republic
Full list of author information is
available at the end of the article

available at the end of the article

\begin{abstract}
In this paper we continue our research from (Monteiro and Trrdý in Discrete Contin. Dyn. Syst. 33(1):283-303, 2013) on continuous dependence on a parameter $k$ of solutions to linear integral equations of the form $x(t)=\widetilde{x}_{k}+\int_{a}^{t} \mathrm{~d}\left[A_{k}\right] x+f_{k}(t)-f_{k}(a)$, $t \in[a, b], k \in \mathbb{N}$, where $-\infty<a<b<\infty, X$ is a Banach space, $L(X)$ is the Banach space of linear bounded operators on $X, \widetilde{x}_{k} \in X, A_{k}:[a, b] \rightarrow L(X)$ have bounded variations on $[a, b], f_{k}:[a, b] \rightarrow X$ are regulated on $[a, b]$. The integrals are understood as the abstract Kurzweil-Stieltjes integral and the studied equations are usually called generalized linear differential equations (in the sense of Kurzweil, cf. (Kurzweil in Czechoslov. Math. J. 7(82):418-449, 1957) or (Kurzweil in Generalized Ordinary Differential Equations: Not Absolutely Continuous Solutions, 2012)). In particular, we are interested in the situation when the variations $\operatorname{var}_{a}^{b} A_{k}$ need not be uniformly bounded. Our main goal here is the extension of Theorem 4.2 from (Monteiro and Trrdý in Discrete Contin. Dyn. Syst. 33(1):283-303, 2013) to the nonhomogeneous case. Applications to second-order systems and to dynamic equations on time scales are included as well.

MSC: Primary 45A05; secondary 34A30; 34N05
\end{abstract}

Keywords: abstract generalized differential equation; continuous dependence; time scale dynamics

\section{Introduction}

In the theory of differential equations it is always desirable to ensure that their solutions depend continuously on the input data. In other words to ensure that small changes of the input data causes also small changes of the corresponding solutions. For ordinary differential equations, in some sense a final result on the continuous dependence was delivered by Kurzweil and Vorel in their paper [1] from 1957. In fact, it was a response to the averaging method introduced few years before by Krasnoselskij and Krein [2]. The extension of the averaging method and the problem of the continuous dependence of solutions on input data were the main motivations for Kurzweil to introduce his notion of generalized differential equations in [3].

\section{空 Springer}

(02014 Monteiro and Tvrdý; licensee Springer. This is an Open Access article distributed under the terms of the Creative Commons Attribution License (http://creativecommons.org/licenses/by/2.0), which permits unrestricted use, distribution, and reproduction in any medium, provided the original work is properly cited. 
By generalized linear differential equations we understand linear integral equations of the form

$$
x(t)=\tilde{x}+\int_{a}^{t} \mathrm{~d}[A] x+f(t)-f(a),
$$

where $-\infty<a<b<\infty, X$ is a Banach space, $L(X)$ is the Banach space of linear bounded operators on $X, \tilde{x} \in X, A:[a, b] \rightarrow L(X)$ has bounded variation on $[a, b], f:[a, b] \rightarrow X$ is regulated on $[a, b]$ and the integrals are understood in the Kurzweil-Stieltjes sense. By a solution of (1.1) we understand a function $x:[a, b] \rightarrow X$ such that $\int_{a}^{b} \mathrm{~d}[A] x$ exists and (1.1) is true for all $t \in[a, b]$.

For $X=\mathbb{R}^{m}$, such equations are special cases of equations introduced in 1957 by Kurzweil (see [3]) in connection with the advanced study of continuous dependence properties of ordinary differential equations (see also [1]). In this connection, we want to highlight the recent monograph [4] bringing a new insight into the topic. Linear equations of the form (1.1) have been in the finite-dimensional case thoroughly treated by Schwabik, Tvrdý and Ashordia (see e.g. [5, 6] and [7]).

Basic theory of the abstract Kurzweil-Stieltjes integral (called also abstract PerronStieltjes or simply gauge-Stieltjes integral) and generalized linear differential equations in a general Banach space has been established by Schwabik in a series of papers [8-10] written between 1996 and 2000. Some of the needed complements have been added in our paper [11].

Taking into account the closing remark in [9], we can see that the following basic existence result is a particular case of [9, Proposition 2.10].

Proposition 1.1 Let $A:[a, b] \rightarrow L(X)$ have a bounded variation on $[a, b]$. Then, (1.1) possesses a unique solution $x$ on $[a, b]$ for every $\tilde{x} \in X$ and every function $f:[a, b] \rightarrow X$ regulated on $[a, b]$ if and only if

$$
\left[I_{X}-\Delta^{-} A(t)\right]^{-1} \in L(X) \text { for all } t \in(a, b]
$$

where $I_{X}$ stands for the identity operator on $X$. In such a case $x$ is regulated on $[a, b], x-f$ has a bounded variation on $[a, b]$ and

$$
\|x(t)\|_{X} \leq c_{A}\left(\|\widetilde{x}\|_{X}+2\|f\|_{\infty}\right) \exp \left(c_{A} \operatorname{var}_{a}^{t} A\right), \quad t \in[a, b],
$$

where $0<c_{A}:=\sup _{t \in(a, b]}\left\|\left[I_{X}-\Delta^{-} A(t)\right]^{-1}\right\|_{L(X)}<\infty$.

Primarily we are concerned with the continuous dependence of solutions of generalized linear differential equations on a parameter. In particular, we assume that the given equation (1.1) has a unique solution $x$ for each $f$ regulated on $[a, b]$ and each $\tilde{x} \in X$ and we consider a sequence of equations depending on a parameter $k \in \mathbb{N}$,

$$
x_{k}(t)=\tilde{x}_{k}+\int_{a}^{t} \mathrm{~d}\left[A_{k}\right] x_{k}+f_{k}(t)-f_{k}(a)
$$

where $A_{k}:[a, b] \rightarrow L(X)$ have bounded variation on $[a, b], f_{k}:[a, b] \rightarrow X$ are regulated on $[a, b]$ and $\widetilde{x}_{k} \in X$ for $k \in \mathbb{N}$. We are looking for conditions ensuring that (1.4) has a unique 
solution $x_{k}$ for each $k$ large enough and the sequence $\left\{x_{k}\right\}$ tends uniformly on $[a, b]$ to $x$, i.e.

$$
\lim _{n \rightarrow \infty}\left\|x_{k}-x\right\|_{\infty}=0
$$

In [12] we proved the following two theorems. The first one deals with the case that the variations of $A_{k}$ are uniformly bounded.

Proposition 1.2 [12, Theorem 3.4] Let $A, A_{k}:[a, b] \rightarrow L(X)$ have bounded variation on $[a, b], f, f_{k}:[a, b] \rightarrow X$ be regulated on $[a, b]$ and $\tilde{x}, \tilde{x}_{k} \in X$ for $k \in \mathbb{N}$. Furthermore, assume (1.2),

$$
\begin{aligned}
& \alpha^{*}:=\sup _{k \in \mathbb{N}}\left(\operatorname{var}_{a}^{b} A_{k}\right)<\infty, \\
& \lim _{k \rightarrow \infty}\left\|A_{k}-A\right\|_{\infty}=0, \\
& \lim _{k \rightarrow \infty}\left\|f_{k}-f\right\|_{\infty}=0, \\
& \lim _{k \rightarrow \infty}\left\|\widetilde{x}_{k}-\widetilde{x}\right\|_{X}=0 .
\end{aligned}
$$

Then (1.1) has a unique solution $x$ on $[a, b]$. Furthermore, for each $k \in \mathbb{N}$ sufficiently large there is a unique solution $x_{k}$ on $[a, b]$ to (1.4) and (1.5) holds.

The second result from [12], inspired by Opial's paper [13], concerns the situation when variations of $A_{k}$ (1.6) need not be uniformly bounded and (1.1) and (1.4) reduce to homogeneous equations.

Proposition 1.3 [12, Theorem 4.2] Let $A, A_{k}:[a, b] \rightarrow L(X)$ have bounded variation on $[a, b]$ and let $\widetilde{x}, \widetilde{x}_{n} \in X$ for $k \in \mathbb{N}$. Furthermore, assume (1.2), (1.9) and

$$
\lim _{k \rightarrow \infty}\left(1+\operatorname{var}_{a}^{b} A_{k}\right)\left\|A_{k}-A\right\|_{\infty}=0 .
$$

Then the equation

$$
x(t)=\widetilde{x}+\int_{a}^{t} \mathrm{~d}[A] x, \quad t \in[a, b],
$$

has a unique solution $x$ on $[a, b]$. Moreover, for each $k \in \mathbb{N}$ sufficiently large, the equation

$$
x_{k}(t)=\widetilde{x}_{k}+\int_{a}^{t} \mathrm{~d}\left[A_{k}\right] x_{k}
$$

has a unique solution $x_{k}$ on $[a, b]$ and (1.5) holds.

Let us recall the following observation.

Lemma 1.4 Let $A, A_{k}:[a, b] \rightarrow L(X)$ have bounded variation on $[a, b]$ and let (1.10) be satisfied. Then (1.7) is true as well.

Proof The proof follows from the obvious inequality

$$
\left\|A_{k}-A\right\|_{\infty} \leq\left(1+\operatorname{var}_{a}^{b} A_{k}\right)\left\|A_{k}-A\right\|_{\infty} \quad \text { for all } k \in \mathbb{N} .
$$


The only known result ( $c f$. [12, Corollary 4.4]) concerning nonhomogeneous equations (1.1), (1.4) and the case when (1.6) is not satisfied requires that $X$ is a finite-dimensional space. The aim of this paper is to fill this gap.

For a more detailed list of related references, see [12].

\section{Preliminaries}

Throughout these notes $X$ is a Banach space and $L(X)$ is the Banach space of bounded linear operators on $X$. By $\|\cdot\|_{X}$ we denote the norm in $X$. Similarly, $\|\cdot\|_{L(X)}$ denotes the usual operator norm in $L(X)$.

Assume that $-\infty<a<b<\infty$ and $[a, b]$ denotes the corresponding closed interval. A set $D=\left\{\alpha_{0}, \alpha_{1}, \ldots, \alpha_{v(D)}\right\} \subset[a, b]$ with $v(D) \in \mathbb{N}$ is said to be a division of $[a, b]$ if $a=\alpha_{0}<\alpha_{1}<$ $\cdots<\alpha_{v(D)}=b$. The set of all divisions of $[a, b]$ is denoted by $\mathcal{D}[a, b]$.

A function $f:[a, b] \rightarrow X$ is called a finite step function on $[a, b]$ if there exists a division $D=\left\{\alpha_{0}, \alpha_{1}, \ldots, \alpha_{v(D)}\right\}$ of $[a, b]$ such that $f$ is constant on every open interval $\left(\alpha_{j-1}, \alpha_{j}\right), j=$ $1,2, \ldots, v(D)$.

For an arbitrary function $f:[a, b] \rightarrow X$ we set $\|f\|_{\infty}=\sup _{t \in[a, b]}\|f(t)\|_{X}$ and

$$
\operatorname{var}_{a}^{b} f=\sup _{D \in \mathcal{D}[a, b]} \sum_{j=1}^{\nu(D)}\left\|f\left(\alpha_{j}\right)-f\left(\alpha_{j-1}\right)\right\|_{X}
$$

is the variation of $f$ over $[a, b]$. If $\operatorname{var}_{a}^{b} f<\infty$, we say that $f$ is a function of bounded variation on $[a, b] . \mathrm{BV}([a, b], X)$ denotes the Banach space of functions $f:[a, b] \rightarrow X$ of bounded variation on $[a, b]$ equipped with the norm $\|f\|_{\mathrm{BV}}=\|f(a)\|_{X}+\operatorname{var}_{a}^{b} f$.

The function $f:[a, b] \rightarrow X$ is called regulated on $[a, b]$ if for each $t \in[a, b)$ there is $f(t+) \in X$ such that $\lim _{s \rightarrow t+}\|f(s)-f(t+)\|_{X}=0$ and for each $t \in(a, b]$ there is $f(t-) \in X$ such that $\lim _{s \rightarrow t-}\|f(s)-f(t-)\|_{X}=0$. By $G([a, b], X)$ we denote the Banach space of regulated functions $f:[a, b] \rightarrow X$ equipped with the norm $\|f\|_{\infty}$. For $t \in[a, b), s \in(a, b]$ we put $\Delta^{+} f(t)=f(t+)-f(t)$ and $\Delta^{-} f(s)=f(s)-f(s-)$. Recall that $\mathrm{BV}([a, b], X) \subset G([a, b], X)$ cf. e.g. the assertion contained in Section 1.5 of [9].

In what follows, by an integral we mean the Kurzweil-Stieltjes integral. Let us recall its definition. As usual, a partition of $[a, b]$ is a tagged system, i.e., a couple $P=(D, \xi)$ where $D=\left\{\alpha_{0}, \alpha_{1}, \ldots, \alpha_{\nu(D)}\right\} \in \mathcal{D}[a, b], \xi=\left(\xi_{1}, \ldots, \xi_{v(D)}\right) \in[a, b]^{\nu(D)}$ and $\alpha_{j-1} \leq \xi_{j} \leq \alpha_{j}$ holds for $j=1,2, \ldots, v(D)$. Furthermore, any positive function $\delta:[a, b] \rightarrow(0, \infty)$ is called a gauge on $[a, b]$. Given a gauge $\delta$ on $[a, b]$, the partition $P$ is called $\delta$-fine if $\left[\alpha_{j-1}, \alpha_{j}\right] \subset$ $\left(\xi_{j}-\delta\left(\xi_{j}\right), \xi_{j}+\delta\left(\xi_{j}\right)\right)$ holds for all $j=1,2, \ldots, \nu(D)$. We remark that for an arbitrary gauge $\delta$ on $[a, b]$ there always exists a $\delta$-fine partition of $[a, b]$. It is stated by the Cousin lemma (see e.g. [5, Lemma 1.4]).

For given functions $F:[a, b] \rightarrow L(X)$ and $g:[a, b] \rightarrow X$ and a partition $P=(D, \xi)$ of $[a, b]$, where $D=\left\{\alpha_{0}, \alpha_{1}, \ldots, \alpha_{v(D)}\right\}, \xi=\left\{\xi_{1}, \ldots, \xi_{v(D)}\right\}$, we define

$$
S(\mathrm{~d} F, g, P)=\sum_{j=1}^{\nu(D)}\left[F\left(\alpha_{j}\right)-F\left(\alpha_{j-1}\right)\right] g\left(\xi_{j}\right) .
$$

We say that $J \in X$ is the Kurzweil-Stieltjes integral (or shortly KS-integral) of $g$ with respect to $F$ on $[a, b]$ and denote $J=\int_{a}^{b} \mathrm{~d}[F] g$ if for every $\varepsilon>0$ there exists a gauge $\delta$ on $[a, b]$ such 
that

$\|S(\mathrm{~d} F, g, P)-J\|_{X}<\varepsilon \quad$ for all $\delta$-fine partitions $P$ of $[a, b]$.

Analogously, we define the integral $\int_{a}^{b} F \mathrm{~d}[g]$ using sums of the form

$$
S(F, \mathrm{~d} g, P)=\sum_{j=1}^{v(D)} F\left(\xi_{j}\right)\left[g\left(\alpha_{j}\right)-g\left(\alpha_{j-1}\right)\right]
$$

Some basic estimates for the KS-integrals are summarized in the following proposition. For the proofs, see [12, Proposition 2.1] and [11, Lemma 2.2].

Proposition 2.1 Let $F:[a, b] \rightarrow L(X)$ and $g:[a, b] \rightarrow X$.

(i) If $F \in \mathrm{BV}([a, b], L(X))$ and $g \in G([a, b], X)$, then $\int_{a}^{b} \mathrm{~d}[F] g$ exists and

$$
\left\|\int_{a}^{b} \mathrm{~d}[F] g\right\|_{X} \leq\left(\operatorname{var}_{a}^{b} F\right)\|g\|_{\infty}
$$

(ii) If $F \in G([a, b], L(X))$ and $g \in \mathrm{BV}([a, b], X)$, then $\int_{a}^{b} \mathrm{~d}[F] g$ exists and

$$
\left\|\int_{a}^{b} \mathrm{~d}[F] g\right\|_{X} \leq 2\|F\|_{\infty}\|g\|_{\mathrm{BV}}
$$

For more details concerning the abstract KS-integration and further references, see [8$10,14]$ and [11].

\section{Main result}

Our main result is based on the following lemma which is an analog of the assertion formulated for ODEs by Kiguradze in [15, Lemma 2.5]. Its variant was used also in the study of FDEs by Hakl, Lomtatidze and Stavrolaukis in [16, Lemma 3.5].

Lemma 3.1 Let $A, A_{k} \in \mathrm{BV}([a, b], L(X))$ for $k \in \mathbb{N}$ and assume that (1.2) and (1.10) hold.

Then there exist $r^{*}>0$ and $k_{0} \in \mathbb{N}$ such that

$$
\begin{aligned}
& \|y\|_{\infty} \leq r^{*}\left(\|y(a)\|_{X}+\left(1+\operatorname{var}_{a}^{b} A_{k}\right) \sup _{t \in[a, b]}\left\|y(t)-y(a)-\int_{a}^{t} \mathrm{~d}\left[A_{k}\right] y\right\|_{X}\right) \\
& \text { for all } y \in G([a, b], X) \text { and } k \geq k_{0} .
\end{aligned}
$$

Proof Assume that (3.1) is not true, i.e. assume that for each $n \in \mathbb{N}$ there are $k_{n} \in \mathbb{N}$ and $y_{n} \in G([a, b], X)$ such that

$$
\left\|y_{n}\right\|_{\infty}>n\left(\left\|y_{n}(a)\right\|_{X}+\left(1+\operatorname{var}_{a}^{b} A_{k_{n}}\right) \sup _{t \in[a, b]}\left\|y_{n}(t)-y_{n}(a)-\int_{a}^{t} \mathrm{~d}\left[A_{k_{n}}\right] y_{n}\right\|_{X}\right) .
$$

We will prove that (3.2) leads to a contradiction. To this aim, first, rewrite inequality (3.2) as

$$
\frac{1}{n}>\left\|u_{n}(a)\right\|_{X}+\left(1+\operatorname{var}_{a}^{b} A_{k_{n}}\right) \sup _{t \in[a, b]}\left\|u_{n}(t)-u_{n}(a)-\int_{a}^{t} \mathrm{~d}\left[A_{k_{n}}\right] u_{n}\right\|_{X},
$$


where

$$
u_{n}(t)=\frac{y_{n}(t)}{\left\|y_{n}\right\|_{\infty}} \quad \text { for } t \in[a, b] \text { and } n \in \mathbb{N} .
$$

Then, by (3.3) and (3.4) we can immediately see that $\left\|u_{n}(a)\right\|_{X}<\frac{1}{n}$ for all $n \in \mathbb{N}$. Hence,

$$
\lim _{n \rightarrow \infty}\left\|u_{n}(a)\right\|_{X}=0
$$

Now, denote

$$
v_{n}(t)=u_{n}(t)-u_{n}(a)-\int_{a}^{t} \mathrm{~d}\left[A_{k_{n}}\right] u_{n} \quad \text { for } t \in[a, b] \text { and } n \in \mathbb{N}
$$

and

$$
z_{n}(t)=u_{n}(t)-v_{n}(t) \quad \text { for } t \in[a, b] \text { and } n \in \mathbb{N} \text {. }
$$

By (3.3) we have

$$
\left.\begin{array}{rl}
\left\|v_{n}\right\|_{\infty} & =\frac{1}{\left\|y_{n}\right\|_{\infty}}\left(\sup _{t \in[a, b]}\left\|y_{n}(t)-y_{n}(a)-\int_{a}^{t} \mathrm{~d}\left[A_{k_{n}}\right] y_{n}\right\|_{X}\right) \\
& <\frac{1}{n\left(1+\operatorname{var}_{a}^{b} A_{k_{n}}\right)} \leq \frac{1}{n} \quad \text { for } n \in \mathbb{N},
\end{array}\right\}
$$

and, in particular,

$$
\lim _{n \rightarrow \infty}\left\|v_{n}\right\|_{\infty}=0 .
$$

Moreover, the equalities (3.6) and (3.7) yield

$$
z_{n}(t)=u_{n}(a)+\int_{a}^{t} \mathrm{~d}\left[A_{k_{n}}\right] u_{n} \quad \text { for } t \in[a, b] \text { and } n \in \mathbb{N} \text {. }
$$

Consequently, $z_{n} \in \mathrm{BV}([a, b], X)$,

$$
z_{n}(a)=u_{n}(a) \quad \text { and } \quad\left\|z_{n}\right\|_{\mathrm{BV}} \leq 1+\operatorname{var}_{a}^{b} A_{k_{n}} \quad \text { for } n \in \mathbb{N} .
$$

Now, let $n \in \mathbb{N}$ be fixed. We have

$$
\begin{aligned}
z_{n}(t) & =z_{n}(a)+\int_{a}^{t} \mathrm{~d}\left[A_{k_{n}}\right] z_{n}+\int_{a}^{t} \mathrm{~d}\left[A_{k_{n}}\right] v_{n} \\
& =z_{n}(a)+\int_{a}^{t} \mathrm{~d}[A] z_{n}+\int_{a}^{t} \mathrm{~d}\left[A_{k_{n}}-A\right] z_{n}+\int_{a}^{t} \mathrm{~d}\left[A_{k_{n}}\right] v_{n} \quad \text { for } t \in[a, b],
\end{aligned}
$$

i.e.

$$
z_{n}(t)=z_{n}(a)+\int_{a}^{t} \mathrm{~d}[A] z_{n}+h_{n}(t) \quad \text { for } t \in[a, b],
$$


where

$$
h_{n}(t)=\int_{a}^{t} \mathrm{~d}\left[A_{k_{n}}-A\right] z_{n}+\int_{a}^{t} \mathrm{~d}\left[A_{k_{n}}\right] v_{n} \quad \text { for } t \in[a, b] .
$$

We claim that

$$
\lim _{n \rightarrow \infty}\left\|h_{n}\right\|_{\infty}=0 .
$$

Indeed, by (3.10) and Proposition 2.1(ii) we have

$$
\begin{aligned}
\sup _{t \in[a, b]}\left\|\int_{a}^{t} \mathrm{~d}\left[A_{k_{n}}-A\right] z_{n}\right\|_{X} & \leq 2\left\|A_{k_{n}}-A\right\|_{\infty}\left\|z_{n}\right\|_{\mathrm{BV}} \\
& \leq 2\left\|A_{k_{n}}-A\right\|_{\infty}\left(1+\operatorname{var}_{a}^{b} A_{k_{n}}\right),
\end{aligned}
$$

wherefrom

$$
\lim _{n \rightarrow \infty} \sup _{t \in[a, b]}\left\|\int_{a}^{t} \mathrm{~d}\left[A_{k_{n}}-\mathcal{A}\right] z_{n}\right\|_{X}=0
$$

follows due to (1.10). Moreover, using Proposition 2.1(i) and (3.8), we get

$$
\sup _{t \in[a, b]}\left\|\int_{a}^{t} \mathrm{~d}\left[A_{k_{n}}\right] v_{n}\right\|_{\infty} \leq\left(\operatorname{var}_{a}^{b} A_{k_{n}}\right)\left\|v_{n}\right\|_{\infty} \leq \frac{1}{n} \frac{\operatorname{var}_{a}^{b} A_{k_{n}}}{\left(1+\operatorname{var}_{a}^{b} A_{k_{n}}\right)} \leq \frac{1}{n}
$$

and, hence,

$$
\lim _{n \rightarrow \infty} \sup _{t \in[a, b]}\left\|\int_{a}^{t} \mathrm{~d}\left[A_{k_{n}}\right] v_{n}\right\|_{X}=0
$$

Now, (3.13) follows immediately from (3.14) and (3.15).

Finally, having in mind Proposition 1.1 (cf. (1.3)) and (3.11), (3.5), and (3.13), we conclude that

$$
\lim _{n \rightarrow \infty}\left\|z_{n}\right\|_{\infty} \leq \lim _{n \rightarrow \infty} c_{A}\left(\left\|z_{n}(a)\right\|_{X}+2\left\|h_{n}\right\|_{\infty}\right) \exp \left(c_{A} \operatorname{var}_{a}^{b} A\right)=0,
$$

i.e.

$$
\lim _{n \rightarrow \infty}\left\|z_{n}\right\|_{\infty}=0 .
$$

This, together with (3.7) and (3.9), implies that $\lim _{n \rightarrow \infty}\left\|u_{n}\right\|_{\infty}=0$, which is impossible as $\left\|u_{n}\right\|_{\infty}=1$ for all $n \in \mathbb{N}$. The assertion of the lemma is true.

Theorem 3.2 Let $A, A_{k} \in \mathrm{BV}([a, b], L(X)), f \in \mathrm{BV}([a, b], X), f_{k} \in G([a, b], X)$ and $\widetilde{x}, \widetilde{x}_{k} \in X$ for $k \in \mathbb{N}$. Assume (1.2), (1.9), (1.10), and

$$
\lim _{k \rightarrow \infty}\left(1+\operatorname{var}_{a}^{b} A_{k}\right)\left(\left\|f_{k}-f\right\|_{\infty}\right)=0 .
$$

Then (1.1) has a unique solution $x \in \mathrm{BV}([a, b], X)$ on $[a, b]$. Moreover, for each $k \in \mathbb{N}$ sufficiently large, (1.4) has a unique solution $x_{k} \in G([a, b], X)$ on $[a, b]$ and $(1.5)$ is true. 
Proof First, recall that, by Lemma 1.4 our assumption (1.10) implies that (1.7) is true, as well. Therefore, by [12, Lemma 4.2], there is $k_{0} \in \mathbb{N}$ such that

$$
\left[I_{X}-\Delta^{-} A_{k}(t)\right]^{-1} \in L(X) \quad \text { for all } t \in(a, b] \text { and } k \geq k_{0}
$$

and (1.4) has a unique solution $x_{k} \in G([a, b], X)$ for each $k \geq k_{0}$ (cf. Proposition 1.1). By Lemma 3.1 we may choose $k_{0} \in \mathbb{N}$ and $r^{*} \in(0, \infty)$ in such way that (3.1) holds.

Put $u_{k}=x_{k}-x$ for $k \geq k_{0}$. Then $u_{k}(a)=\tilde{x}_{k}-\tilde{x}$ and

$$
u_{k}(t)-\int_{a}^{t} \mathrm{~d}\left[A_{k}\right] u_{k}-\left(\widetilde{x}_{k}-\widetilde{x}\right)=\int_{a}^{t} \mathrm{~d}\left[A_{k}-A\right] x+\left(f_{k}(t)-f(t)\right)-\left(f_{k}(a)-f(a)\right)
$$

for $t \in[a, b]$ and $k \geq k_{0}$. Using (3.1) we deduce that the inequality

$$
\left\|u_{k}\right\|_{\infty} \leq r^{*}\left(\left\|\widetilde{x}_{k}-\widetilde{x}\right\|_{X}+\left(1+\operatorname{var}_{a}^{b} A_{k}\right)\left(2\left\|A_{k}-A\right\|_{\infty}\|x\|_{\mathrm{BV}}+2\left\|f_{k}-f\right\|_{\infty}\right)\right)
$$

holds for all $k \geq k_{0}$. Thus, due to (1.9), (1.10) and (3.17), we have $\lim _{k \rightarrow \infty}\left\|u_{k}\right\|_{\infty}=0$, wherefrom (1.5) immediately follows. The proof of the theorem has been completed.

Remark 3.3 The proof of Theorem 3.2 could be substantially simplified and also extended to the case $f \in G([a, b], X)$ if the following assertion was true.

Let $A, A_{k} \in \mathrm{BV}([a, b], L(X))$ for $k \in \mathbb{N}$ and

$$
\lim _{k \rightarrow \infty}\left(1+\operatorname{var}_{a}^{b} A_{k}\right)\left\|A_{k}-A\right\|_{\infty}=0 .
$$

Then

$$
\lim _{k \rightarrow \infty}\left(\sup _{t \in[a, b]}\left\|\int_{a}^{t} \mathrm{~d}\left[A_{k}\right] f-\int_{a}^{t} \mathrm{~d}[A] f\right\|_{X}\right)=0
$$

holds for each $f \in G([a, b], X)$.

Unfortunately, this is in general not true even in the scalar case as shown by the following example that was communicated to us by Ivo Vrkoč.

Example 3.4 Let $[a, b]=[0,1]$. For $k \in \mathbb{N}$ put $^{\mathrm{a}}$

$$
\begin{aligned}
& n_{k}=\left[k^{3 / 2}\right]+1, \quad \tau_{m, k}=\frac{1}{2^{n_{k}-m}} \quad \text { if } m \in\left\{0,1, \ldots, n_{k}\right\}, \\
& a_{0, k}=\frac{2^{n_{k}}}{k}(-1)^{n_{k}}, \quad b_{0, k}=\frac{1}{k}(-1)^{n_{k}-1}, \\
& a_{m, k}=\frac{2^{n_{k}-m+1}}{k}(-1)^{n_{k}-m}, \quad b_{m, k}=\frac{3}{k}(-1)^{n_{k}-m+1} \quad \text { if } m \in\left\{1,2, \ldots, n_{k}-1\right\}
\end{aligned}
$$

and define

$$
A_{k}(t)= \begin{cases}0 & \text { if } t \in\left[0, \tau_{0, k}\right], \\ a_{m, k} t+b_{m, k} & \text { if } t \in\left[\tau_{m, k}, \tau_{m+1, k}\right] \text { and } m \in\left\{0,1, \ldots, n_{k}-1\right\}\end{cases}
$$


and

$$
A(t)=0 \quad \text { for } t \in[0,1] \text {. }
$$

It is easy to verify that

$$
\operatorname{var}_{0}^{1} A_{k} \leq \frac{1}{k}+\frac{2\left(n_{k}-1\right)}{k} \leq \frac{1}{k}+2 \sqrt{k}<\infty
$$

and

$$
\left(1+\operatorname{var}_{0}^{1} A_{k}\right)\left\|A_{k}-A\right\|_{\infty} \leq\left(1+\frac{2 n_{k}-1}{k}\right) \frac{1}{k} \leq \frac{1}{k}+\frac{2}{\sqrt{k}}+\frac{1}{k^{2}}
$$

for all $k \in \mathbb{N}$. In particular, (3.18) is true. However, if

$$
f(t)= \begin{cases}\frac{(-1)^{n}}{\sqrt[4]{n}} & \text { if } t \in\left(2^{-n}, 2^{-(n-1)}\right] \text { for some } n \in \mathbb{N} \\ 0 & \text { if } t=0\end{cases}
$$

then $f$ is regulated, $\operatorname{var}_{0}^{1} f=\infty$ and (3.19) is not valid since

$$
\int_{0}^{1} \mathrm{~d}\left[A_{k}\right] f \geq \frac{2}{k} \sum_{m=1}^{n_{k}-1} \frac{1}{\sqrt[4]{m}}>\frac{2}{k} \int_{1}^{n_{k}} \frac{1}{\sqrt[4]{t}} \mathrm{~d} t=\frac{8}{3 k}\left(\sqrt[4]{\left(n_{k}\right)^{3}}-1\right)
$$

where the right-hand side evidently tends to $\infty$ for $k \rightarrow \infty$.

Moreover, the functions (3.20) and (3.21) provide us with the argument explaining that the condition $f \in \mathrm{BV}([a, b], X)$ in Theorem 3.2 cannot be extended to $f \in G([a, b], X)$. Indeed, consider the equations

$$
x(t)=\int_{0}^{t} \mathrm{~d}[A] x+f(t), \quad t \in[0,1]
$$

and

$$
x_{k}(t)=\int_{0}^{t} \mathrm{~d}\left[A_{k}\right] x_{k}+f_{k}(t), \quad t \in[0,1], k \in \mathbb{N},
$$

where $f_{k}(t)=f(t)$ for $t \in[0,1]$ and $k \in \mathbb{N}$. Obviously, $x=f$ is a solution to (3.23) on $[0,1]$ and, for any $k \in \mathbb{N},(3.24)$ possesses a solution $x_{k}$ on $[0,1]$. Furthermore, conditions (1.10) and (3.17) are satisfied. However, as we will see, $x_{k}$ does not converge to $x$.

Let $k \in \mathbb{N}$ be fixed. It is not difficult to verify that the solution to $(3.24)$ on $[0,1]$ is given by

$$
x_{k}(t)= \begin{cases}f(t) & \text { if } t \in\left[0, \tau_{0, k}\right], \\ c_{m, k} \exp \left(a_{m, k} t\right) & \text { if } t \in\left(\tau_{m, k}, \tau_{m+1, k}\right] \text { and } m \in\left\{0,1, \ldots, n_{k}-1\right\},\end{cases}
$$

where

$$
c_{0, k}=f\left(\tau_{1, k}\right) \exp \left(-a_{0, k} \tau_{0, k}\right)=f\left(\tau_{1, k}\right) \exp \left(\frac{1}{k}(-1)^{n_{k}+1}\right)
$$


and

$$
c_{m, k}=c_{m-1, k} \exp \left(\left(a_{m-1, k}-a_{m, k}\right) \tau_{m, k}\right)+\left(f\left(\tau_{m+1, k}\right)-f\left(\tau_{m, k}\right)\right) \exp \left(-a_{m, k} \tau_{m, k}\right)
$$

for $m=1, \ldots, n_{k}-1$. Furthermore, since

$$
\left(a_{0, k}-a_{1, k}\right) \tau_{1, k}=\frac{4}{k}(-1)^{n_{k}} \quad \text { and } \quad a_{1, k} \tau_{1, k}=-\frac{2}{k}(-1)^{n_{k}}
$$

we have

$$
c_{1, k}=c_{0, k} \exp \left(\frac{4}{k}(-1)^{n_{k}}\right)+\left(f\left(\tau_{2, k}\right)-f\left(\tau_{1, k}\right)\right) \exp \left(\frac{2}{k}(-1)^{n_{k}}\right) .
$$

Similarly, for $m=2,3, \ldots, n_{k}-1$ we have

$$
\left(a_{m-1, k}-a_{m, k}\right) \tau_{m, k}=\frac{6}{k}(-1)^{n_{k}-m+1} \quad \text { and } \quad \tau_{m, k} a_{m, k}=\frac{2}{k}(-1)^{n_{k}-m}
$$

and hence

$$
\begin{aligned}
c_{m, k}= & c_{m-1, k} \exp \left(\frac{6}{k}(-1)^{n_{k}-m+1}\right) \\
& +\left(f\left(\tau_{m+1, k}\right)-f\left(\tau_{m, k}\right)\right) \exp \left(\frac{2}{k}(-1)^{n_{k}-m+1}\right) .
\end{aligned}
$$

From these formulas we can deduce that

$$
\begin{aligned}
c_{m, k}= & \exp \left(\frac{2}{k}(-1)^{n_{k}+1}\right)\left(c_{0, k}+\sum_{j=2}^{m+1}(-1)^{j+1} f\left(\tau_{j, k}\right)\right) \\
& +\exp \left(\frac{4}{k}(-1)^{n_{k}+1}\right) \sum_{j=1}^{m}(-1)^{j} f\left(\tau_{j, k}\right)
\end{aligned}
$$

if $m$ is even, while for $m$ odd and $m>1$ we get

$$
\begin{aligned}
c_{m, k}= & \exp \left(\frac{4}{k}(-1)^{n_{k}}\right)\left(c_{0, k}+\sum_{j=2}^{m}(-1)^{j+1} f\left(\tau_{j, k}\right)\right) \\
& +\exp \left(\frac{2}{k}(-1)^{n_{k}}\right) \sum_{j=1}^{m+1}(-1)^{j} f\left(\tau_{j, k}\right) .
\end{aligned}
$$

In particular, $x_{k}(1)=c_{n_{k}-1, k} \exp (-4 / k)$ for $k \in \mathbb{N}$. Using the above relations and the definition of $f$, we get

$$
\begin{aligned}
c_{n_{k}-1, k}= & \exp \left(\frac{4}{k}\right)\left(c_{0, k}+\sum_{j=2}^{n_{k}-1}(-1)^{j+1} \frac{(-1)^{n_{k}-j+1}}{\sqrt[4]{n_{k}-j+1}}\right) \\
& +\exp \left(\frac{2}{k}\right) \sum_{j=1}^{n_{k}}(-1)^{j} \frac{(-1)^{n_{k}-j+1}}{\sqrt[4]{n_{k}-j+1}}
\end{aligned}
$$




$$
\begin{aligned}
= & \exp \left(\frac{4}{k}\right)\left(c_{0, k}+\sum_{m=2}^{n_{k}-1} \frac{1}{\sqrt[4]{m}}\right)-\exp \left(\frac{2}{k}\right) \sum_{m=1}^{n_{k}} \frac{1}{\sqrt[4]{m}} \\
= & \exp \left(\frac{2}{k}\right)\left(c_{0, k} \exp \left(\frac{2}{k}\right)-1\right)+\exp \left(\frac{2}{k}\right)\left(\exp \left(\frac{2}{k}\right)-1\right) \sum_{m=2}^{n_{k}-1} \frac{1}{\sqrt[4]{m}} \\
& -\exp \left(\frac{2}{k}\right) \frac{1}{\sqrt[4]{n_{k}}}
\end{aligned}
$$

if $n_{k}$ is even, and

$$
\begin{aligned}
c_{n_{k}-1, k} & =\exp \left(\frac{2}{k}\right)\left(c_{0, k}-\sum_{m=1}^{n_{k}-1} \frac{1}{\sqrt[4]{m}}\right)+\exp \left(\frac{4}{k}\right) \sum_{m=2}^{n_{k}} \frac{1}{\sqrt[4]{m}} \\
& =\exp \left(\frac{2}{k}\right)\left(c_{0, k}-1\right)+\exp \left(\frac{2}{k}\right)\left(\exp \left(\frac{2}{k}\right)-1\right) \sum_{m=2}^{n_{k}-1} \frac{1}{\sqrt[4]{m}}+\exp \left(\frac{4}{k}\right) \frac{1}{\sqrt[4]{n_{k}}}
\end{aligned}
$$

if $n_{k}$ is odd.

Clearly, $\lim _{k \rightarrow \infty} c_{0, k}=0$,

$$
\lim _{k \rightarrow \infty} \exp \left(\frac{2}{k}\right)\left(c_{0, k} \exp \left(\frac{2}{k}\right)-1\right)=\lim _{k \rightarrow \infty} \exp \left(\frac{2}{k}\right)\left(c_{0, k}-1\right)=-1
$$

and

$$
\lim _{k \rightarrow \infty} \exp \left(\frac{2}{k}\right) \frac{1}{\sqrt[4]{n_{k}}}=\lim _{k \rightarrow \infty} \exp \left(\frac{4}{k}\right) \frac{1}{\sqrt[4]{n_{k}}}=0
$$

On the other hand, like in (3.22), we have

$$
\begin{aligned}
\exp (2 / k)(\exp (2 / k)-1) \sum_{m=2}^{n_{k}-1} \frac{1}{\sqrt[4]{m}} & =\exp (2 / k) \frac{\exp (2 / k)-1}{2 / k} \frac{2}{k} \sum_{m=2}^{n_{k}-1} \frac{1}{\sqrt[4]{m}} \\
& >\exp (2 / k) \frac{\exp (2 / k)-1}{2 / k} \frac{2}{k} \int_{2}^{n_{k}} \frac{1}{\sqrt[4]{t}} \mathrm{~d} t \\
& =\exp (2 / k) \frac{\exp (2 / k)-1}{2 / k} \frac{8}{3 k}\left(\sqrt[4]{\left(n_{k}\right)^{3}}-\sqrt[4]{2^{3}}\right),
\end{aligned}
$$

where the right-hand side tends to $\infty$ when $k \rightarrow \infty$. Consequently, the sequence $x_{k}(1)$ cannot have a finite limit for $k \rightarrow \infty$.

Remark 3.5 Reasonable examples of sequences $\left\{f_{k}\right\} \subset G([a, b], X)$ that tend to a function $f$ of bounded variation are provided $e$.g. by sequences of the form $f_{k}=g_{k}+h_{k}$, where $\left\{g_{k}\right\} \subset$ $\mathrm{BV}([a, b], X)$ tends to $f \in \mathrm{BV}([a, b], X)$ and $\left\{h_{k}\right\} \subset G([a, b], X)$ tends to 0 .

Remark 3.6 For $F:[a, b] \rightarrow L(X)$ and $D=\left\{\alpha_{0}, \alpha_{1}, \ldots, \alpha_{m}\right\} \in \mathcal{D}[a, b]$, define

$$
V_{a}^{b}(F, D)=\sup \left\{\left\|\sum_{j=1}^{m}\left[F\left(\alpha_{j}\right)-F\left(\alpha_{j-1}\right)\right] y_{j}\right\|_{X}: y_{j} \in X,\left\|y_{j}\right\|_{X} \leq 1, j=1,2, \ldots, m\right\}
$$


and

$$
\mathrm{SV}_{a}^{b}(F)=\sup \left\{V_{a}^{b}(F, D) ; D \in \mathcal{D}[a, b]\right\}
$$

Then $\mathrm{SV}_{a}^{b}(F)$ is said to be the semi-variation of $F$ on $[a, b](c f . e . g . ~[17]) .^{\mathrm{b}}$ It is clear that if $F \in \mathrm{BV}([a, b], L(X))$ then $F$ has bounded semi-variation on $[a, b]$ while the reversed implication is not true in general (cf. [18, Theorem 2]). By [8] and [11], the Kurzweil-Stieltjes integral $\int_{a}^{b} \mathrm{~d}[A] x$ is well defined when both functions, $A$ and $x$, are regulated and $A$ has bounded semi-variation. Therefore, the study of generalized linear differential equations has a good sense also when $A$ is regulated and has bounded semi-variation instead of having $A \in \mathrm{BV}([a, b], X), c f$. [9] and [10]. However, the possible extension of Theorem 3.1 to such a case remains open.

Analogously to operator valued functions, the semi-variation of a function $f:[a, b] \rightarrow X$ could be defined using

$$
\begin{aligned}
& V_{a}^{b}(f, D) \\
& \quad=\sup \left\{\left\|\sum_{j=1}^{m} F_{j}\left[f\left(\alpha_{j}\right)-f\left(\alpha_{j-1}\right)\right]\right\|_{X}: F_{j} \in L(X),\left\|F_{j}\right\|_{L(X)} \leq 1, j=1,2, \ldots, m\right\} .
\end{aligned}
$$

However, it may be shown that, in this case, $f$ has a bounded semi-variation if and only $f \in \mathrm{BV}([a, b], X)$. Therefore, the possible replacement of the condition $f \in \mathrm{BV}([a, b], X)$ in Theorem 3.1 by the requirement that $f$ has a bounded semi-variation is not interesting.

\section{Some applications}

\section{Second-order measure equations}

Let $Y$ be a Banach space, $\tilde{y}, \tilde{z} \in Y, P, Q \in \mathrm{BV}([a, b], L(Y))$ and $g, h \in \mathrm{BV}([a, b], Y)$. Consider the following system of generalized linear differential equations:

$$
\left.\begin{array}{ll}
y(t)=\tilde{y}+\int_{a}^{t} \mathrm{~d}[P] z+g(t)-g(a), & t \in[a, b], \\
z(t)=\widetilde{z}+\int_{a}^{t} \mathrm{~d}[Q] y+h(t)-h(a), & t \in[a, b] .
\end{array}\right\}
$$

Put $X=Y \times Y$ and $\|(y, z)\|_{X}=\|y\|_{Y}+\|z\|_{Y}$ for $(y, z) \in X$ and define functions $A:[a, b] \rightarrow$ $L(X)$ and $f:[a, b] \rightarrow X$ by

$$
\begin{gathered}
A(t)(y, z)=(P(t) z, Q(t) y) \in X \quad \text { and } \quad f(t)=(g(t), h(t)) \in X \\
\quad \text { for } y, z \in Y \text { and } t \in[a, b] .
\end{gathered}
$$

Clearly,

$$
\|A(t)\|_{L(X)}=\|P(t)\|_{L(Y)}+\|Q(t)\|_{L(Y)} \quad \text { for } t \in[a, b], \quad \operatorname{var}_{a}^{b} A \leq \operatorname{var}_{a}^{b} P+\operatorname{var}_{a}^{b} Q
$$

and system (4.1) can be reformulated as $(1.1)$, where $\tilde{x}=(\tilde{y}, \tilde{z})$ and $x=(y, z)$ is a function with values in $X$. One can verify that condition (1.2) is satisfied whenever one of the following 
conditions is true:

$$
\begin{aligned}
& {\left[I_{Y}-\Delta^{-} Q(t) \Delta^{-} P(t)\right]^{-1} \in L(Y) \quad \text { for } t \in(a, b],} \\
& {\left[I_{Y}-y \Delta^{-} P(t) \Delta^{-} Q(t)\right]^{-1} \in L(Y) \quad \text { for } t \in(a, b],}
\end{aligned}
$$

where $I_{Y}$ stands for the identity operator on $Y$.

Indeed, assume e.g. that (4.3) holds and let $\left[I_{X}-\Delta^{-} A(t)\right] x=0$ for some $x=(y, z) \in X$ and $t \in(a, b]$. Then

$$
y-\Delta^{-} P(t) z=0 \quad \text { and } \quad z-\Delta^{-} Q(t) y=0,
$$

i.e.

$$
y=\Delta^{-} P(t) z \quad \text { and } \quad\left[I_{Y}-\Delta^{-} Q(t) \Delta^{-} P(t)\right] z=0 .
$$

By (4.3) the latter equality can happen only if $z=0$. Consequently $y=0$, and hence $x=0$, as well. Similarly, we would show that $\left[I_{X}-\Delta^{-} A(t)\right] x=0$ implies $x=0$ also in the case that (4.4) is satisfied. This shows that the operator $I_{X}-\Delta^{-} A(t)$ is injective.

To prove its surjectivity, assume first (4.3) and let $(u, v) \in X$ be given. Put

$$
z=\left(\left[I_{Y}-\Delta^{-} Q(t) \Delta^{-} P(t)\right]^{-1}\right)\left(v+\Delta^{-} Q(t) u\right) \quad \text { and } \quad y=u+\Delta^{-} P(t) z .
$$

Then, $y-\Delta^{-} P(t) z=u$ and

$$
\begin{aligned}
z-\Delta^{-} Q(t) y & =z-\Delta^{-} Q(t) \Delta^{-} P(t) z-\Delta^{-} Q(t) u \\
& =\left[I_{Y}-\Delta^{-} Q(t) \Delta^{-} P(t)\right] z-\Delta^{-} Q(t) u=v,
\end{aligned}
$$

that is, $\left[I_{X}-\Delta^{-} A(t)\right] x=(u, v)$ for $x=(y, z)$. Similarly, we can show that for each $(u, v) \in X$ there is $x \in X$ such that $\left[I_{X}-\Delta^{-} A(t)\right] x=(u, v)$ also in the case that (4.4) is satisfied. The operator $I_{X}-\Delta^{-} A(t)$ is surjective. To summarize, according to the Banach theorem, the operator $I_{X}-\Delta^{-} A(t)$ possesses a bounded $\left[I_{X}-\Delta^{-} A(t)\right]^{-1}$.

Now, consider the systems

$$
\left.\begin{array}{ll}
y_{k}(t)=\tilde{y}_{k}+\int_{a}^{t} \mathrm{~d}\left[P_{k}\right] z_{k}+g_{k}(t)-g_{k}(a), & t \in[a, b], k \in \mathbb{N}, \\
z_{k}(t)=\widetilde{z}_{k}+\int_{a}^{t} \mathrm{~d}\left[Q_{k}\right] y_{k}+h_{k}(t)-h_{k}(a), & t \in[a, b], k \in \mathbb{N},
\end{array}\right\}
$$

where $\widetilde{y}_{k}, \widetilde{z}_{k} \in Y, P_{k}, Q_{k} \in B V([a, b], L(Y)), g_{k}, h_{k} \in G([a, b], Y)$ and $k \in \mathbb{N}$. Assume that (4.3) or (4.4) is true and

$$
\begin{aligned}
& \lim _{k \rightarrow \infty}\left\|\widetilde{y}_{k}-\tilde{y}\right\|_{Y}=0, \quad \lim _{k \rightarrow \infty}\left\|\widetilde{z}_{k}-\tilde{z}\right\|_{Y}=0, \\
& \lim _{k \rightarrow \infty}\left(1+\operatorname{var}_{a}^{b} P_{k}+\operatorname{var}_{a}^{b} Q_{k}\right)\left(\left\|P_{k}-P\right\|_{\infty}+\left\|Q_{k}-Q\right\|_{\infty}\right)=0
\end{aligned}
$$


and

$$
\lim _{k \rightarrow \infty}\left(1+\operatorname{var}_{a}^{b} P_{k}+\operatorname{var}_{a}^{b} Q_{k}\right)\left(\left\|g_{k}-g\right\|_{\infty}+\left\|h_{k}-h\right\|_{\infty}\right)=0
$$

Define $A_{k}:[a, b] \rightarrow L(X)$ and $f_{k}:[a, b] \rightarrow X$ for $k \in \mathbb{N}$ like $A$ and $f$ in (4.2) (however, replace $P, Q, g$, and $h$ by $P_{k}, Q_{k}, g_{k}$ and $h_{k}$, respectively). It is easy to see that then the assumptions of Theorem 3.2 are satisfied. Therefore, we can state the following assertion.

Corollary 4.1 Assume that (4.3) or (4.4) holds and that (4.8)-(4.10) are satisfied. Then system (4.1) has a unique solution $(y, z) \in \mathrm{BV}([a, b], Y \times Y)$ on $[a, b]$. Moreover, for each $k \in \mathbb{N}$ sufficiently large, the system (4.7) has a unique solution $\left(y_{k}, z_{k}\right) \in G([a, b], Y \times Y)$ on $[a, b]$ and

$$
\lim _{k \rightarrow \infty}\left(\left\|y_{k}-y\right\|_{\infty}+\left\|z_{k}-z\right\|_{\infty}\right)=0
$$

In [19], Meng and Zhang investigated the continuous dependence on a parameter $k$ for second-order linear measure differential equations of the form

$$
\mathrm{d} y^{\bullet}+\mathrm{d}\left[\mu_{k}(t)\right] y=0, \quad t \in[0,1], \quad y(0)=\tilde{y}, \quad y^{\bullet}(0)=\widetilde{z}, \quad k \in \mathbb{N},
$$

where $\mu_{k}$ are normalized measures on $[0,1]$ (generated by functions of bounded variation on $[0,1]$ and right-continuous in $(0,1)), \tilde{y}, \widetilde{z} \in \mathbb{R}$ and $y^{\bullet}$ stands for the generalized rightderivative of $y$. The main result of [19] is Theorem 1.1, which states that the weak* convergence $\mu_{k} \rightarrow \mu$ implies the uniform convergence $y_{k} \rightrightarrows y$ of the corresponding solutions, the weak* convergence $y_{k}^{\bullet} \rightarrow y^{\bullet}$ and the ending velocity convergence $y_{k}^{\bullet}(1) \rightarrow y^{\bullet}(1)$.

Notice that our systems (4.7) reduce to (4.11) when $[a, b]=[0,1], X=\mathbb{R}, P_{k}(t)=t$ and $Q_{k}(t)=\mu_{k}(t)$ for $t \in[0,1]$ and both $g_{k}$ and $h_{k}$ are constant [19, Definition 3.1]. Similarly, if, in addition, $P(t)=t$ and $Q(t)=\mu(t)$ for $t \in[0,1]$ and both $g$ and $h$ are constant, then system (4.1) reduces to the second-order linear measure differential equation of the form

$$
\mathrm{d} y^{\bullet}+\mathrm{d}[\mu(t)] y=0, \quad t \in[0,1], \quad y(0)=\tilde{y}, \quad y^{\bullet}(0)=\widetilde{z}, \quad k \in \mathbb{N},
$$

where $\mu$ is a normalized measure on $[0,1]$ and $\tilde{y}, \tilde{z} \in \mathbb{R}$. Obviously, both existence conditions (4.3) and (4.4) are now satisfied. In view of this, assuming that $\mu$ and $\mu_{k}$ have a bounded variation on $[0,1]$ and

$$
\lim _{k \rightarrow \infty}\left(1+\operatorname{var}_{0}^{1} \mu_{k}\right)\left\|\mu_{k}-\mu\right\|_{\infty}=0,
$$

it follows from our Corollary 4.1 that

$$
\lim _{k \rightarrow \infty}\left(\left\|y_{k}-y\right\|_{\infty}+\left\|y_{k}^{\bullet}-y^{\bullet}\right\|_{\infty}\right)=0
$$

holds for the corresponding solutions of (4.11) and (4.12).

Thus, in comparison with Theorem 1.1 in [19], our convergence assumptions are partially stronger. The reason is that our result includes also the uniform convergence of the sequence $\left\{y_{k}^{\bullet}\right\}$. On the other hand, the weak* convergence which appears in [19] includes the uniform boundedness of the variations $\operatorname{var}_{0}^{1} \mu_{k}$ (cf. e.g. [20, Lemma 2.4] or [21, Section 26]) which is not required in our case. 


\section{Linear dynamic equations on time scales}

Let us recall some basics of the theory of dynamic equations on time scales. A nonempty closed subset $\mathbb{T}$ of $\mathbb{R}$ is called time scale. For given $a, b \in \mathbb{T}$, we put $[a, b]_{\mathbb{T}}=[a, b] \cap \mathbb{T}$. For $t \in \mathbb{T}$, we define

$$
\rho(t):=\sup [a, t) \cap \mathbb{T} \quad \text { and } \quad \sigma(t):=\inf (t, b] \cap \mathbb{T} .
$$

The point $t \in \mathbb{T}$ is said to be right-dense if $\sigma(t)=t$, while it is left-dense if $\rho(t)=t$. A function $f:[a, b]_{\mathbb{T}} \rightarrow \mathbb{R}^{m}$ is $r d$-continuous in $[a, b]_{\mathbb{T}}$ if $f$ is continuous at every right-dense point of $[a, b]_{\mathbb{T}}$ and there exists $f(t-)$ for every left-dense point $t \in[a, b]_{\mathbb{T}}$ (see e.g. [22]).

Let us consider the linear dynamic equation

$$
y^{\Delta}(t)=P(t) y(t)+h(t), \quad y(a)=\tilde{y}, \quad t \in[a, b]_{\mathbb{T}},
$$

where $\tilde{y} \in \mathbb{R}^{m}$ and $P:[a, b]_{\mathbb{T}} \rightarrow L\left(\mathbb{R}^{m}\right), h:[a, b]_{\mathbb{T}} \rightarrow \mathbb{R}^{m}$ are rd-continuous functions and $y^{\Delta}$ stands for the $\Delta$-derivative. By a solution of (4.13) we understand a function $y:[a, b]_{\mathbb{T}} \rightarrow \mathbb{R}^{m}$ satisfying the integral equation

$$
y(t)=\tilde{y}+\int_{a}^{t}[P(s) y(s)+h(s)] \Delta s, \quad t \in[a, b]_{\mathbb{T}},
$$

where the integral is the Riemann $\Delta$-integral defined e.g. in [22].

As noticed by Slavík (see [23, Theorem 5]), the Riemann $\Delta$-integral can be regarded as a special case of the Kurzweil-Stieltjes integral. More precisely:

Let $f:[a, b]_{\mathbb{T}} \rightarrow \mathbb{R}^{m}$ be an rd-continuous function and

$$
\begin{aligned}
& \tilde{\sigma}(t):=\inf [t, b] \cap \mathbb{T} \text { for } t \in[a, b], \\
& F_{1}(t)=\int_{a}^{t} f(s) \Delta s \quad \text { for } t \in[a, b]_{\mathbb{T}}
\end{aligned}
$$

and

$$
F_{2}(t)=\int_{a}^{t} f(\widetilde{\sigma}(s)) \mathrm{d}[\widetilde{\sigma}(s)] \text { for } t \in[a, b] .
$$

Then $F_{2}(t)=F_{1}(\widetilde{\sigma}(t))$ holds for $t \in[a, b]$.

As a consequence, a relationship between the solutions of (4.13) and generalized linear differential equations can be deduced.

Proposition 4.2 [23, Theorem 12] If $y:[a, b]_{\mathbb{T}} \rightarrow \mathbb{R}^{m}$ is a solution of (4.13) then

$$
x=y \circ \tilde{\sigma} y:[a, b] \rightarrow \mathbb{R}^{m}
$$

is a solution of (1.1), where

$$
\left.\begin{array}{l}
A(t)=\int_{a}^{t} P(\widetilde{\sigma}(s)) \mathrm{d}[\tilde{\sigma}(s)] \quad \text { for } t \in[a, b] \quad \text { and } \\
f(t)=\int_{a}^{t} h(\widetilde{\sigma}(s)) \mathrm{d}[\widetilde{\sigma}(s)] \quad \text { for } t \in[a, b] .
\end{array}\right\}
$$


Symmetrically, if $x:[a, b] \rightarrow \mathbb{R}^{m}$ is a solution of (1.1), with $A$ and $f$ given by (4.14), then $y:[a, b]_{\mathbb{T}} \rightarrow \mathbb{R}^{m}$ defined by $y(t)=x(t)$ for $t \in[a, b]_{\mathbb{T}}$ is a solution of (4.13).

It is important to mention that, thanks to the properties of $\widetilde{\sigma}:[a, b] \rightarrow[a, b]_{\mathbb{T}}$, the functions $A:[a, b] \rightarrow L\left(\mathbb{R}^{n}\right)$ and $f:[a, b] \rightarrow \mathbb{R}^{n}$ given by (4.14) are well defined, left-continuous and of bounded variation on $[a, b]$.

Using the correspondence stated in Proposition 4.2 and Theorem 3.2 we obtain the following result.

Theorem 4.3 Let $P, P_{k}:[a, b]_{\mathbb{T}} \rightarrow L\left(\mathbb{R}^{m}\right), h, h_{k}:[a, b]_{\mathbb{T}} \rightarrow \mathbb{R}^{m}$ for $k \in \mathbb{N}$ be rd-continuous functions in $[a, b]_{\mathbb{T}}$ and let $\tilde{y}, \tilde{y}_{k} \in \mathbb{R}^{m}, k \in \mathbb{N}$, be given. Assume that

$$
\left.\begin{array}{l}
\lim _{k \rightarrow \infty}\left\|\tilde{y}_{k}-\tilde{y}\right\|_{\mathbb{R}^{m}}=0, \\
\lim _{k \rightarrow \infty}\left[1+\sup _{t \in[a, b]_{\mathbb{T}}}\left\|P_{k}(t)\right\|_{L\left(\mathbb{R}^{m}\right)}\right] \sup _{t \in[a, b]_{\mathbb{T}}}\left\|\int_{a}^{t}\left(P_{k}(s)-P(s)\right) \Delta s\right\|_{L\left(\mathbb{R}^{m}\right)}=0, \\
\lim _{k \rightarrow \infty}\left[1+\sup _{t \in[a, b]_{\mathbb{T}}}\left\|P_{k}(t)\right\|_{L\left(\mathbb{R}^{m}\right)}\right] \sup _{t \in[a, b]_{\mathbb{T}}}\left\|\int_{a}^{t}\left(h_{k}(s)-h(s)\right) \Delta s\right\|_{\mathbb{R}^{m}}=0 .
\end{array}\right\}
$$

Then initial value problem (4.13) has a solution $y$, the initial value problems

$$
y_{k}^{\Delta}(t)=P_{k}(t) y_{k}(t)+h_{k}(t), \quad y_{k}(a)=\widetilde{y}_{k}, \quad t \in[a, b]_{\mathbb{T}}
$$

have solutions $y_{k}$ for all $k \in \mathbb{N}$, and

$$
\lim _{k \rightarrow \infty} \sup _{t \in[a, b]_{\mathbb{T}}}\left\|y_{k}(t)-y(t)\right\|_{\mathbb{R}^{m}}=0 .
$$

Proof For each $k \in \mathbb{N}$ and $t \in[a, b]$, define

$$
A_{k}(t)=\int_{a}^{t} P_{k}(\widetilde{\sigma}(s)) \mathrm{d}[\widetilde{\sigma}(s)] \text { and } f_{k}(t)=\int_{a}^{t} h_{k}(\widetilde{\sigma}(s)) \mathrm{d}[\widetilde{\sigma}(s)]
$$

It is not difficult to see that, if $a \leq c<d \leq b$, then

$$
\left\|A_{k}(d)-A_{k}(c)\right\|_{L\left(\mathbb{R}^{m}\right)}=\left\|\int_{c}^{d} P_{k}(\widetilde{\sigma}(s)) \mathrm{d}[\widetilde{\sigma}(s)]\right\|_{L\left(\mathbb{R}^{m}\right)} \leq\left(\sup _{t \in[a, b]]_{\mathbb{T}}}\left\|P_{k}(t)\right\|_{L\left(\mathbb{R}^{m}\right)}\right)\left(\operatorname{var}_{c}^{d} \widetilde{\sigma}\right),
$$

and, consequently,

$$
\operatorname{var}_{a}^{b} A_{k} \leq\left(\sup _{t \in[a, b]_{\mathbb{T}}}\left\|P_{k}(t)\right\|_{L\left(\mathbb{R}^{m}\right)}\right)\left(\operatorname{var}_{a}^{b} \tilde{\sigma}\right), \quad k \in \mathbb{N} .
$$

On the other hand,

$$
\left\|A_{k}-A\right\|_{\infty}=\sup _{t \in[a, b]}\left\|\int_{a}^{\widetilde{\sigma}(t)}\left(P_{k}(s)-P(s)\right) \Delta s\right\|_{L\left(\mathbb{R}^{m}\right)} \leq \sup _{t \in[a, b]_{\mathbb{T}}}\left\|\int_{a}^{t}\left(P_{k}(s)-P(s)\right) \Delta s\right\|_{L\left(\mathbb{R}^{m}\right)}
$$

and, analogously,

$$
\left\|f_{k}-f\right\|_{\infty} \leq \sup _{t \in[a, b]_{\mathbb{T}}}\left\|\int_{a}^{t}\left(h_{k}(s)-h(s)\right) \Delta s\right\|_{\mathbb{R}^{m}} .
$$


These estimates, together with (4.15) and (4.16) imply that the assumptions of Theorem 3.2 are satisfied. Therefore, the uniform convergence of solutions $x_{k}$ of equation (1.5) to the solution $x$ of (1.1) follows. Since by Proposition 4.2 the solutions of (4.13) and (4.17) are, respectively, obtained as the restriction of $x$ and $x_{k}$ to $[a, b]_{\mathbb{T}}$, the proof is complete.

Remark 4.4 It is worth to mention that Theorem 4.3 given above encompasses Theorem 5.5 from [12]. This is due to the fact that the weighted convergence assumptions in [12, Theorem 5.5] involves not only the supremum $\sup _{t \in[a, b]_{\mathbb{T}}}\left\|P_{k}(t)\right\|_{L\left(\mathbb{R}^{m}\right)}$, but also $\sup _{t \in[a, b]_{\mathbb{T}}}\left\|h_{k}(t)\right\|_{\mathbb{R}^{m}}$.

\section{Competing interests}

The authors declare that they have no competing interests.

Authors' contributions

The authors contributed equally to the manuscript and read and approved the final draft.

\section{Author details}

${ }^{1}$ Mathematical Institute, Academy of Sciences of Czech Republic, Žitná 25, Praha, 115 67, Czech Republic. ${ }^{2}$ Mathematical Institute, Academy of Sciences of Czech Republic, Žitná 25, Praha, 115 67, Czech Republic.

\section{Acknowledgements}

GA Monteiro has bee supported by the Institutional Research Plan No. AV0Z10190503 and by the Academic Human Resource Program of the Academy of Sciences of the Czech Republic and M Tvrdý has been supported by the grant No. 14-06958S of the Grant Agency of the Czech Republic and by the Institutional Research Plan No. AV0Z10190503. The authors sincerely thank Ivo Vrkoč for his valuable contribution to this paper.

\section{Endnotes}

a $[x]$ stands, as usual, for the integer part of the nonnegative real number $x$.

b Sometimes it is called also the $\mathcal{B}$-variation of $F$ on $[a, b]$ (with respect to the bilinear triple $\mathcal{B}=(L(X), X, X), c f$. e.g. $[8]$ ).

Received: 20 January 2014 Accepted: 13 March 2014 Published: 26 Mar 2014

\section{References}

1. Kurzweil, J, Vorel, Z: Continuous dependence of solutions of differential equations on a parameter. Czechoslov. Math. J. 7(82), 568-583 (1957)

2. Krasnoselskij, MA, Krein, SG: On the averaging principle in nonlinear mechanics. Usp. Mat. Nauk 10(3), 147-152 (1955) (in Russian)

3. Kurzweil, J: Generalized ordinary differential equation and continuous dependence on a parameter. Czechoslov. Math. J. 7(82), 418-449 (1957)

4. Kurzweil, J: Generalized Ordinary Differential Equations: Not Absolutely Continuous Solutions. Series in Real Analysis, vol. 11. World Scientific, Singapore (2012)

5. Schwabik, Š: Generalized Ordinary Differential Equations. World Scientific, Singapore (1992)

6. Schwabik, Š, Tvrdý, M, Vejvoda, O: Differential and Integral Equations: Boundary Value Problems and Adjoints. Academia, Praha (1979)

7. Ashordia, M: On the correctness of linear boundary value problems for systems of generalized ordinary differential equations. Proc. Georgian Acad. Sci., Math. 1, 385-394 (1993)

8. Schwabik, Š: Abstract Perron-Stieltjes integral. Math. Bohem. 121, 425-447 (1996)

9. Schwabik, Š: Linear Stieltjes integral equations in Banach spaces. Math. Bohem. 124, 433-457 (1999)

10. Schwabik, Š: Linear Stieltjes integral equations in Banach spaces II. Operator valued solutions. Math. Bohem. 125, 431-454 (2000)

11. Monteiro, GA, Tvrdý, M: On Kurzweil-Stieltjes integral in Banach space. Math. Bohem. 137, 365-381 (2012)

12. Monteiro, GA, Tvrdý, M: Generalized linear differential equations in a Banach space: continuous dependence on a parameter. Discrete Contin. Dyn. Syst. 33(1), 283-303 (2013). doi:10.3934/dcds.2013.33.283

13. Opial, Z: Continuous parameter dependence in linear systems of differential equations. J. Differ. Equ. 3, 571-579 (1967)

14. Dudley, RM, Norvaiša, R: Concrete Functional Calculus. Springer Monographs in Mathematics. Springer, New York (2011)

15. Kiguradze, IT: Boundary value problems for systems of ordinary differential equations. Itogi Nauki Tekh., Ser. Sovrem. Probl. Mat., Noveishie Dostizh. 30, 3-103 (1987) (in Russian). English transl.: J. Sov. Math. 43, 2259-2339 (1988)

16. Hakl, R, Lomtatidze, A, Stavrolaukis, IP: On a boundary value problem for scalar linear functional differential equations. Abstr. Appl. Anal. 2004(1), 45-67 (2004). doi:10.1155/S1085337504309061

17. Hönig, CS: Volterra Stieltjes-Integral Equations. Mathematics Studies, vol. 16. Elsevier, Amsterdam (1975)

18. Thorp, BLD: On the equivalence of certain notions of bounded variation. J. Lond. Math. Soc. 43, $247-252$ (1968) 
19. Meng, G, Zhang, M: Dependence of solutions and eigenvalues of measure differential equations on measures. J. Differ. Equ. 254, 2196-2232 (2013)

20. Meng, G, Zhang, M: Measure differential equations I. Continuity of solutions in measures with weak* topology. Preprint, Tsinghua University (2009)

21. Glivenko, VI: The Stieltjes Integral. ONTI, Moscow (1936) (in Russian)

22. Bohner, M, Peterson, A: Dynamic Equations on Time Scales: An Introduction with Applications. Birkhäuser, Boston (2001)

23. Slavík, A: Dynamic equations on time scales and generalized ordinary differential equations. J. Math. Anal. Appl. 385 534-550 (2012)

10.1186/1687-2770-2014-71

Cite this article as: Monteiro and Tvrdý: Continuous dependence of solutions of abstract generalized linear differential equations with potential converging uniformly with a weight. Boundary Value Problems 2014, $2014: 71$

Submit your manuscript to a SpringerOpen ${ }^{\circ}$ journal and benefit from:

- Convenient online submission

- Rigorous peer review

- Immediate publication on acceptance

- Open access: articles freely available online

- High visibility within the field

- Retaining the copyright to your article

Submit your next manuscript at $>$ springeropen.com 